\title{
A pilot study on vision related quality of life deter- mined using the NEI RQL-42 questionnaire
}

\section{WDH Gillan*}

\author{
Department of Optometry, University of Johannesburg, PO Box 524, Auckland Park, 2006 South \\ Africa
}

<wgillan@uj.ac.za>

Received 14 April 2009; Revised version accepted 1 September 2009

\section{Introduction}

A common measure of sight used by eye care practitioners is Snellen visual acuity. Newer measures of sight include contrast sensitivity function and the ETDRS visual acuity chart. Visual acuity, and to a lesser extent contrast sensitivity function, are limited indicators of how an individual actually sees. What does it mean, in terms of how an individual experiences his or her world, when a visual acuity is recorded as $3 / 60$ ? Or perhaps when the measure of sight is recorded as "sees fingers at 1 metre"? What does it mean from a quality-of-life point of view, when an individual with $6 / 7.5$ visual acuity (VA) states that their sight is hazy all the time or that they cannot drive at night due to glare created by oncoming traffic? Just how do such acuities influence the daily life of individuals unfortunate enough to be in such a predicament? Interacting with many keratoconics perhaps forces one to attempt to make sense of how such a patient experiences their world visually and how their medical condition influences their life. Giving a personal insight into being keratoconic, a young individual stated that "he wished he was a ten dioptre myope" and that "myopes are lucky to not be keratoconic". How does a post-LASIK or radial keratotomy state influence quality-of-life? Does VA give adequate insight to these questions?

The RAND corporation (a non-profit organization whose mission is to improve policy and decision making through research and analysis, operating since 1948: see www.rand.org) in association with the National Eye Institute (sponsoring institute) started the development of a "refractive error quality-oflife" questionnaire (NEI RQL-42) in 1997 (Hays RD. Development of the National Eye Institute refractive error quality of life instrument (NEI-RQL-42). See Hays@rand.org). The development and validation of this instrument was reported in 2003.1,2 The NEI RQL-42 is a self administered questionnaire consisting of 42 questions that were designed to assess how refractive error affects day to day life $^{2}$. The 42 questions are then arranged to assess 13 scales of visuallyrelated quality of life (clarity of vision, expectations, near vision, far vision, diurnal fluctuations, activity limitations, glare, symptoms, dependence on correction, worry, sub-optimal correction, appearance and satisfaction with correction) $)^{2}$. Further investigation into the reliability and validity of the NEI RQL-42 instrument was conducted by Nichols et $a l^{3}$, where it was found that the NEI RQL-42 instrument gives good reliability and validity. The scoring and administration of the questionnaire are explained in a user's manual ${ }^{14}$. Essentially the subject answers 42 questions by marking a box corresponding to their response. All questions are scored so that higher scores indicate better quality-of-life (from $0 \%$ to $100 \%$ ). Once the subject has completed the questionnaire, items within each scale are averaged to create the 13 visually related quality-of-life scales (see above) ${ }^{4}$. A set of norms 
was determined when the initial reliability and validity study was conducted, investigating 665 myopes, 375 hyperopes and 114 emmetropes. ${ }^{1,2,4}$ The norms for the 13 scales can be seen in Table 1 .

The NEI RQ1-42 has been used on numerous occasions to evaluate the influence of various conditions on visual quality-of-life. The effects of overnight corneal reshaping on visual quality-of-life were investigated making use of the NEI RQL-42 survey $^{5-7}$. The studies found that some scales of the survey improved when soft contact lenses were worn (clarity of vision and glare) while other scales showed an improvement following the overnight wear of lenses (activity limitations, symptoms and dependence on refractive correction). The treatment of contact lens related dry eye using cyclosporine was evaluated using the NEI RQL-42 questionnaire ${ }^{8}$. The results of this study revealed no significant difference (in all 13 scales) between the placebo and experimental groups. Visual performance and patient satisfaction were assessed in presbyopic subjects wearing a multifocal soft contact lens and/or the monovision technique ${ }^{9}$. Having used the RQL-42 in this study, the authors concluded that the multifocal lens was preferred by the majority of subjects. Investigating vision-related quality-of-life, the NEI RQL-42 questionnaire was used to evaluate the difference between Synergeyes and soft toric contact lenses. The results of the study showed that toric soft contact lenses produced less symptoms of dryness, itching, and lens awareness. On the other hand Synergeyes lenses resulted in better visual acuity ${ }^{10}$.

The aim of this pilot study was multi-faceted. Firstly the results of this study would be compared to the norms that were developed previously when the instrument was evaluated for validity and reliability ${ }^{4}$. Secondly, software that was developed specifically for this study would be used and evaluated. Lastly, the results of two post-radial keratotomy (RK) patients and one keratoconic patient were compared to the norms determined from the remaining subjects.

\section{Method}

All subjects volunteered to complete the questionnaire once the purpose of the investigation was explained to them. All subjects remained anonymous (except for those that chose to include their names on the questionnaire). All subjects were treated according to the tenets of the Declaration of Helsinki. The majority of subjects were final year optometry students at the University of Johannesburg (including the single keratoconic). Two post-RK patients from the optometry clinic at the University of Johannesburg were asked to volunteer to complete the questionnaire. The data from each questionnaire were entered into a computer operating a program specially developed to analyze and compile the data (The program was written and developed by Professor A Rubin of the Optometry Department: University of Johannesburg). The analysis of the data was conducted according to the method suggested by Hays and Spritzer ${ }^{4}$. Simply, each question in the questionnaire is designed to assess one aspect of the 13 scales alluded to above. The appropriate response to each question is assigned to the relevant scale and a mean value is generated (see Table 1 as an example). In some instances (for example the satisfaction with correction scale) only one question relates while other scales may have up to seven question responses being used to develop the scale (for example symptoms) ${ }^{4}$. A total of 22 subjects completed the questionnaire, one keratoconic, two post-RK subjects and nineteen emmetropic, myopic and hyperopic subjects.

Table 1. Mean measures for a sample of 665 myopes, 375 hyperopes and 114 emmetropes (in percentage and standard deviation) for the thirteen scales as determined by Hays and Spritzer ${ }^{4}$. The higher the score the better the quality-of-life.

\begin{tabular}{|l|l|l|}
\hline Measure & Mean & Standard deviation \\
\hline Clarity of vision & 83.85 & 18.36 \\
Expectations & 43.57 & 38.22 \\
Near vision & 83.94 & 18.03 \\
Far vision & 83.48 & 15.85 \\
Diurnal variations & 74.58 & 23.13 \\
Activity limitations & 85.28 & 21.92 \\
Glare & 76.40 & 26.41 \\
Symptoms & 79.20 & 16.79 \\
Dependence of correction & 42.38 & 34.75 \\
Worry & 61.31 & 26.04 \\
Suboptimal correction & 92.74 & 17.28 \\
Appearance & 79.31 & 27.00 \\
Satisfaction with correction & 74.85 & 22.55 \\
\hline
\end{tabular}




\section{Results}

Figure 1 shows box and whisker plots for the 19 emmetropic, myopic and hyperopic subjects. For each quality-of-life scale the box indicates the mean while the whiskers show the mean \pm one standard deviation. The thirteen visual scales are indicated along the $x$-axis and percentage score on the $y$-axis (remember: the higher the score the better the visually related quality-of-life).

The plot (Figure 1) gives a visual indication of how the non-keratoconic/RK group experiences their visual quality-of-life. It will also be noted that the majority of scores are close to, or above, $50 \%$.

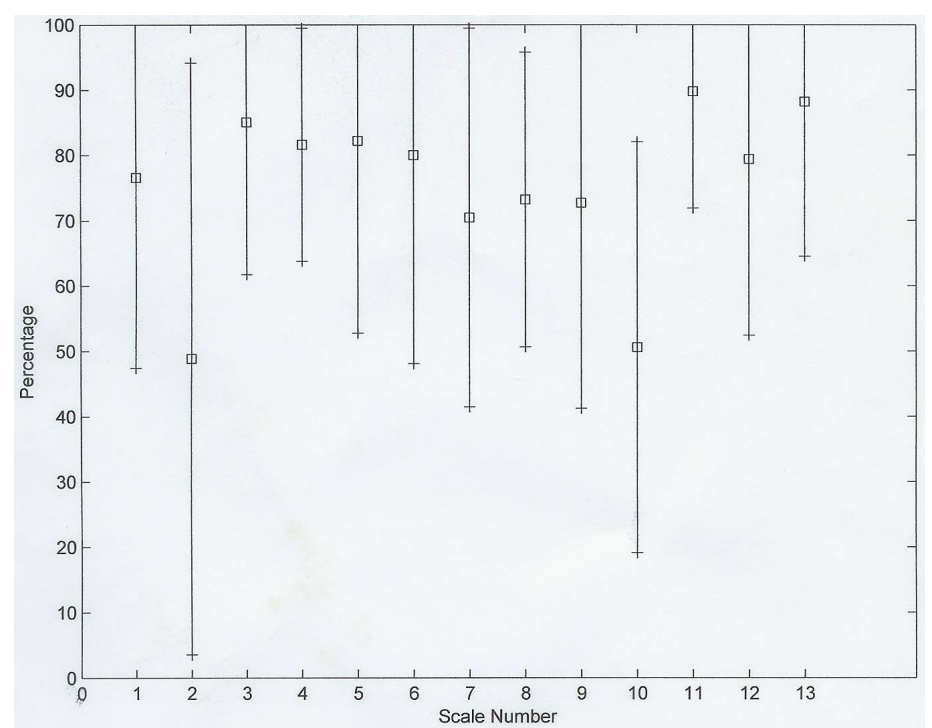

Figure 1. RQL scores for myopic, hyperopic and emmetropic subjects $(n=19)$ are shown. Percentage scores are shown on the $y$-axis and the 13 scales of the NEI RQL questionnaire on the $x$-axis. Boxes indicate means and whiskers the standard deviations.
Table 2 gives the development norms ${ }^{4}$ and the results from this study for the thirteen scales for the 19 non-keratoconic/RK subjects (second and third columns respectively). Any differences between the development norms and the results of this study can be seen. No hypothesis tests were done as the complete data set of the development norms are not available. It will also be noticed that only three visual scales are separated by more than ten percentage points (ten percentage points being an arbitrary number), namely dependence on correction, worry and satisfaction with correction.

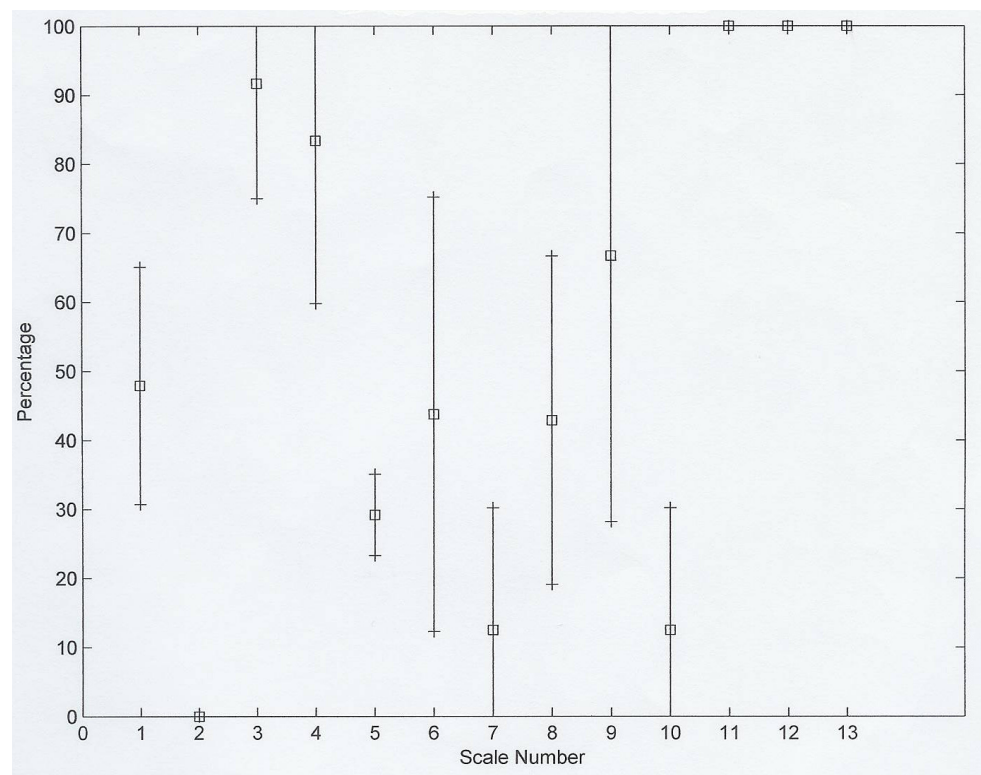

Figure 2. RQL scores for a single keratoconic subject is shown. Percentage scores are shown on the $y$-axis and the 13 scales of the NEI RQL questionnaire on the $x$-axis. Boxes indicate means and whiskers the standard deviations.

Table 2. Mean scores for the thirteen scales for the data determined by Hays and Spritzer ${ }^{4}$ are shown in the second column. The scores for the 19 non-keratoconic/RK subjects, the keratoconic subject and the two post-RK subjects are provided in columns 3 , 4 and 5 respectively. Data collected in this study have been rounded to the nearest number. Standard deviations are given in parentheses.

\begin{tabular}{|l|l|l|l|l|}
\hline Measure & Norms & Emm/My/Hy & Keratoconic & RK \\
\hline Clarity of vision & $83.85(18.36)$ & $77(29)$ & $47(17)$ & $21(9)$ \\
Expectations & $43.57(38.22)$ & $49(45)$ & $0(0)$ & $0(0)$ \\
Near vision & $83.94(18.03)$ & $85(23)$ & $92(17)$ & $21(9)$ \\
Far vision & $83.48(15.85)$ & $82(18)$ & $83(24)$ & $42(29)$ \\
Diurnal variations & $74.58(23.13)$ & $82(29)$ & $29(6)$ & $12(18)$ \\
Activity limitations & $85.28(21.92)$ & $80(32)$ & $44(31)$ & $0(0)$ \\
Glare & $76.40(26.41)$ & $70(29)$ & $12(18)$ & $12(18)$ \\
Symptoms & $79.20(16.79)$ & $73(23)$ & $43(24)$ & $73(37)$ \\
Dependence on correction & $42.38(34.75)$ & $73(32)$ & $67(38)$ & $17(0)$ \\
Worry & $61.31(26.06)$ & $51(32)$ & $12(18)$ & $25(36)$ \\
Suboptimal correction & $92.74(17.28)$ & $90(18)$ & $100(0)$ & $75(35)$ \\
Appearance & $79.31(27.00)$ & $79(27)$ & $100(0)$ & $33(28)$ \\
Satisfaction with correction & $74.85(22.55)$ & $88(24)$ & $100(0)$ & $40(57)$ \\
\hline
\end{tabular}


Figure 2 presents a box and whisker plot of the data collected from the single keratoconic subject. A comparison of Figures 1 and 2 shows the difference between the perceptions of the non-keratoconic/ RK group and the keratoconic subject as it relates to visual quality-of-life. The scores for the thirteen vision scales for the keratoconic subject can be seen in Table 2 (column 4). Figure 3 details the mean data for the two post-RK subjects. It is immediately apparent that these two subjects have a reduced visual quality-of-life (according to this survey). The data can be compared directly in Table 2 . Figure 4 shows the mean data for the three groups of subjects in this study collectively, allowing for an easier comparison to be made between the three groups.

\section{Discussion}

The aim of this study was to compare the norms developed for the NEI RQL ${ }^{1-2,4}$ with the data collected from subjects who participated in the present investigation, to utilize and evaluate software developed to analyze this data and to get an indication of how keratoconus and RK might affect the refractive quality-of-life of individuals who live with these two conditions.

Table 2 shows the scores for the 13 scales evaluated by the NEI RQL questionnaire as obtained by RAND investigators ${ }^{1-2,4}$ as well as the scores of the subjects who participated in this study. No statistical analysis was performed on these data as the complete data set, as obtained from the RAND investigators, is not available.

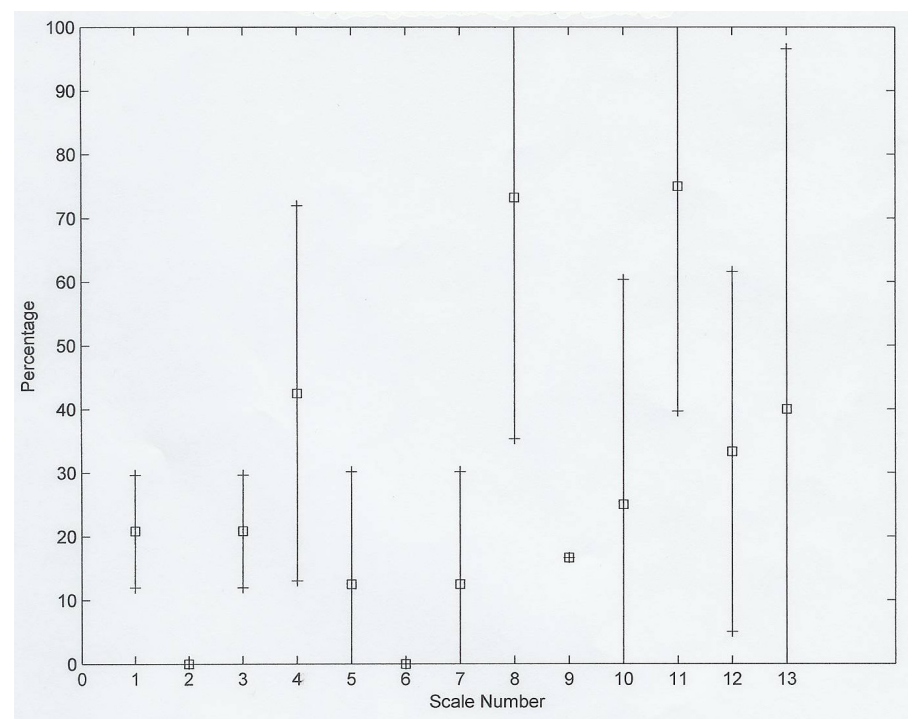

Figure 3. RQL scores for two post-radial keratotomy subjects are shown. Percentage scores are shown on the $y$-axis and the 13 scales of the NEI RQL questionnaire on the $x$-axis. Boxes indicate means and whiskers the standard deviations.

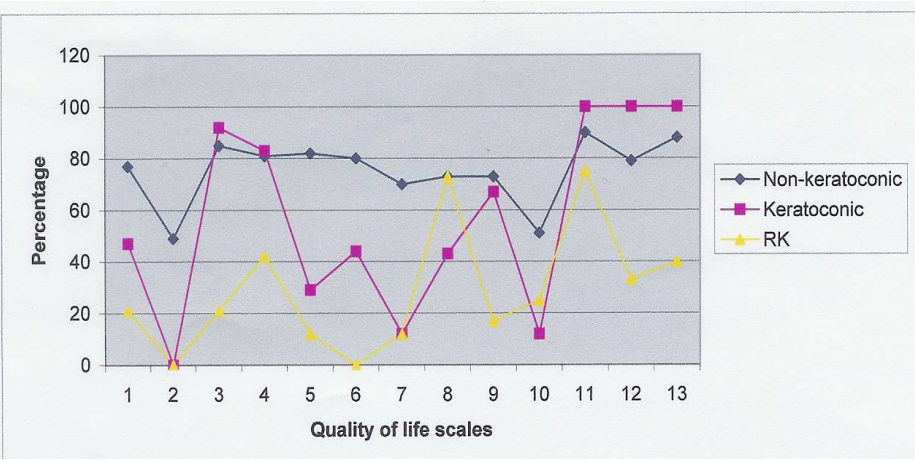

Figure 4. Particulars for all three groups of subjects are shown using colored profiles. It is clear that the post-radial keratotomy subjects (yellow profile) scored worst (overall) in this particular study. It should be kept in mind, however, that subject numbers varied across groups.

The scores of the 19 non-keratoconic/RK subjects compare reasonably favorably with the norms obtained by the originators of the questionnaire (compare columns 2 and 3). The scores obtained from the 19 non-keratoconic/RK subjects suggest that the visually related quality-of-life for these subjects is "not too bad". A limitation of this study was to combine data from emmetropic, myopic and hyperopic subjects, however, the data were combined so that a more direct comparison could be made with the data presented by the RAND investigators ${ }^{1-2,4}$. Further investigation is needed where emmetropes can be compared with other refractive groups more directly (of course this would mean that one assumes that emmetropes have a "better" visually related quality-of-life). The data collected from the keratoconic subject (see Table 2, column 4 and Figure 2) shows that this subject's visually related quality-of-life is lower (or worse) than the 19 non-keratoconic/RK subjects. How much worse is still a concept that is difficult to appreciate without being keratoconic. An interesting observation, however, is that this keratoconic subject rates the scales for appearance and satisfaction with correction as 100 which suggests that this subject feels that the refractive compensation that he is presently using (a piggy back system) is the best that he is going to get. The scale for sub-optimal correction is also rated as 100 . This scale evaluates how often an individual wears a device to look better (in terms of appearance) even though the device is uncomfortable or not as effective as another device might be. A score of 100 shows that the device is never worn to look better. Figure 4 shows this trend clearly. Data for the two post-radial 
keratotomy subjects is shown in column 5 of Table 2. These two subjects rate their visually related qualityof-life as being the worst of all the subjects that participated in this study. It is, however, interesting that the aided VA of one subject was 6/12 right and left eye while the other subject's unaided VA as R 6/7.5 and $\mathrm{L} 6 / 9$. One might be inclined to think that a VA of $6 / 12$ or $6 / 9$ might not be too bad, yet these two subjects perceive their visually related quality-of-life as being the poorest. Another interesting observation concerning one of the post-RK subjects was the fact that, even though her scores were amongst the lowest of any subject in this study, she stated the following on the questionnaire: "Die operasie was die beste ding in my lewe" (The operation was the best thing in my life). It is difficult to reconcile a statement such as this with the fact that this subject's visually related quality-of- life questionnaire responses scored the worst of all subjects in the study.

The NEI RQL-42 questionnaire gives one a different insight into the effects of refractive abnormalities on the visually related quality-of-life of our patients, however, it is still not complete. It is most likely that a practitioner will never truly understand what it is like to be keratoconic or a post-refractive surgery patient, for whom things have not gone as wished, without having the condition themselves. Nevertheless, making use of an instrument like the NEI RQL-42 questionnaire will augment other measures of sight that are used (VA, contrast sensitivity) in an attempt to understand the effects of refractive condition on vision function.

\section{References}

1. Berry S, Mangione CM, Lindblad AS, McDonnell PJ, NEIRQL focus group investigators. Development of the National Eye Institute refractive error quality of life questionnaire: Focus groups. Ophthalmol 2003110 2285-2291.

2. Hays RD, Mangione CM, Ellwein L, Lindblad AS, Spritzer KL, McDonnell PJ, NEI-RQL research group. Psychometric properties of the National Eye Institute refractive error quality of life instrument. Ophthalmol $20031102292-$ 2301.

3. Nichos JJ, Mitchell L, Saracino M, Zadnik K. Reliability and validity of refractive error-specific quality-of-life instruments. Arch Ophthalmol 2003121 1289-1296.

4. Hays RD, Spritzer KL. National Eye Institute refractive error quality of life instrument (NEI RQ1-42) version 1.0. A manual for use and scoring. Los Angeles. 2002.

5. Lipson MJ, Sugar A, Musch DC. Overnight corneal reshaping versus soft daily wear: a visual quality of life study (interim results). Eye Contact Lens 200430 214-217.
6. Lipson MJ, Sugar A, Musch DC. Overnight corneal reshaping versus soft disposable lenses: vision-related quality-oflife differences from a randomized clinical trial. Optom Vis Sci 200582 886-891.

7. Berntsen DA, Mitchell GL, Barr JT. The effect of overnight contact lens corneal reshaping on refractive error specific quality of life. Optom Vis Sci 200683 354-359.

8. Willen K, McGwin G, Liu B, Owsley C, Rosenstiel C. Efficacy of cyclosporine $0.05 \%$ ophthalmic emulsion in contact lens wearers with dry eyes. Eye Contact Lens 200834 43-45.

9. Richdale K, Mitchell GL, Zadnik K. Comparison of multifocal and monovision soft contact lens corrections in patients with astigmatic presbyopia. Optom Vis Sci 200683 266-273.

10. Lipson MJ, Musch DC. Synergeyes versus soft toric lenses: vision related quality of life. Optom Vis Sci 200784 593597. 